\title{
BMJ Open Epidemiology of obesity and overweight in sub-Saharan Africa: a protocol for a systematic review and meta-analysis
}

\author{
Sibhatu Biadgilign, ${ }^{1}$ Tennyson Mgutshini, ${ }^{1}$ Demewoz Haile, ${ }^{2}$ \\ Bereket Gebremichael, ${ }^{3}$ Yonatan Moges, ${ }^{4}$ Kelemu Tilahun ${ }^{5}$
}

To cite: Biadgilign S, Mgutshini T, Haile D, et al. Epidemiology of obesity and overweight in subSaharan Africa: a protocol for a systematic review and meta-analysis. BMJ Open 2017;7:e017666. doi:10.1136/ bmjopen-2017-017666

- Prepublication history and additional materials for this paper are available online. To view these files, please visit the journal online (http://dx.doi. org/10.1136/bmjopen-2017017666).

Received 8 May 2017 Revised 28 September 2017 Accepted 18 0ctober 2017

\section{CrossMark}

${ }^{1}$ Department of Health Studies, College of Human Science, University of South Africa, Pretoria, South Africa

${ }^{2}$ School of Public Health, College of Health Sciences, Addis Ababa University, Addis Ababa, Ethiopia ${ }^{3}$ Department of Nursing and Midwifery, Allied School of Health Science, Addis Ababa University, Addis Ababa, Ethiopia ${ }^{4}$ School of Medicine and Public Health, University of Newcastle, Newcastle, Australia

${ }^{5}$ Department of Public Health, College of Medical and Health Science, Wollega University, Nekemte, Orimia, Ethiopia

Correspondence to Dr Sibhatu Biadgilign; sibhatu2010@gmail.com

\section{ABSTRACT}

Introduction Globally, overweight and obesity were estimated to cause 3.4 million deaths, $3.9 \%$ of years of life lost and $3.8 \%$ of disability-adjusted life years in 2010. Despite the fact that obesity and overweight is a problem of high-income countries, low- and middleincome countries (LMICs), in particular urban settings of sub-Saharan African countries, face the challenge of an increasing trend. The aim of this systematic review and meta-analysis will be to determine the prevalence of obesity and overweight individuals in sub-Saharan Africa and to help guide policy planners in the decision-making process for the increase in non-communicable diseases in Africa.

Methods and analyses A comprehensive systematic review and meta-analysis of published studies on the prevalence of obesity and overweight in sub-Saharan Africa will be conducted. A computerised internet search using Medline/PubMed, Google Scholar and EMBASE databases and reference lists of previous prevalence studies and detailed search strategy and cross-checking of reference lists of published peer-reviewed articles will be conducted to identify all epidemiological and/ or clinical studies published in English and French. We will use the PRISMA (Preferred Reporting Items for Systematic reviews and Meta-Analyses) statement for reporting. The overall effect (pooled estimated effect size) of the prevalence of obesity and overweight will be analysed using the Der Simonian-Laird random effects meta-analysis (random effects model) and the obesity proportion (with $95 \% \mathrm{Cl}$ ) will be measured.

Ethics and dissemination The underlying work is based on systematic reviews of published data and thus doed not require ethical review approval. The findings of the systematic review will be disseminated in different conferences and seminars and will be published in a reputable and refereed international peer-reviewed journal.

PROSPERO registration number CRD42017064942.

\section{INTRODUCTION}

Globally, overweight and obesity were estimated to cause 3.4 million deaths, $3.9 \%$ of years of life lost and $3.8 \%$ of disability-adjusted life
Strengths and limitations of this study

- A comprehensive systematic review and metaanalysis of published studies on the prevalence of obesity and overweight in sub-Saharan Africa will be conducted.

- All epidemiological studies of observational (crosssectional, cohort, case-control) and randomised controlled trials will be included.

- A wide range of studies and settings will be included.

- Studies with a small sample size ( $<30$ participants) and those conducted outside sub-Saharan Africa will be excluded.

years in $2010 .{ }^{1}$ Obesity and overweight cause serious metabolic disorders such as diabetes, cardiovascular disease, hypertension and cancer. ${ }^{2}$ A rapid rise in obesity and being overweight due to nutrition alteration (fast food) and sedentary lifestyles have affected society health in an unprecedented way. Changes in urbanisation, westernisation of diets, lifestyle modifications and the consumption of a highly processed diet contribute to the rise in obesity, decreased physical activity and increasing risk of metabolic and cardiovascular diseases. ${ }^{3}$ In the same way, considerable demographic, social and economic changes have been happening in low- and middle-income countries (LMICs) including rapid urbanisation, expanded education, increased industrialisation and rising incomes in the past few decades. ${ }^{2}$

A systematic analysis of global data on the prevalence of obesity and overweight showed that it has risen significantly over the past three decades. ${ }^{1}$

Despite the fact that obesity and overweight is a problem of high-income countries, LMICs-particularly in urban settings and sub-Saharan African countries-face the challenge of an increasing trend. ${ }^{4}$ Apparently, 
in 2014 more than 1.9 billion adults aged 18 years and older were overweight. Similarly, $13 \%$ of the world's adult population ( $11 \%$ of men and $15 \%$ of women) were obese in 2014. ${ }^{4}$ In Africa, the number of children who are overweight or obese has nearly doubled from 5.4 million in 1990 to 10.6 million in $2014 .^{4}$ The clear picture of obesity and overweight is that it can affect a child's immediate health, educational attainment and quality of life. ${ }^{5}$ Evidence shows that a high body mass index (BMI) in childhood is associated with an increased risk of cardiovascular disease, type 2 diabetes mellitus ${ }^{67}$ and hypertension $^{7}$ and contributes to obesity in adulthood. ${ }^{9}$ Establishing monitoring systems can provide evidence of the impact and effectiveness of interventions in reducing the prevalence of obesity ${ }^{5}$ and can be used to inform policy and practice decisions on how to prevent and treat childhood obesity. ${ }^{10}$ There is a dearth of epidemiological information on comprehensive evidence in sub-Saharan African countries on the estimation of obesity and those who are overweight. The aim of this systematic review and meta-analysis study will be to determine the prevalence of obesity and overweight in individuals in sub-Saharan Africa in order to provide guidance to policy planners for decision-making processes for the increase in non-communicable diseases in Africa.

\section{METHODS}

\section{Study design and data source}

A comprehensive systematic review and meta-analysis of published studies on the prevalence of obesity and overweight in sub-Saharan Africa will be conducted. A computerised internet search using Web of Science, Medline/ PubMed, Scopus, Excerpta Medica Database Guide (EMBASE) and Google Scholar databases and reference lists of previous prevalence studies based on a detailed search strategy including the DHS Programme website (www.measuredhs.com) and cross-checking of reference lists of published peer-reviewed articles will be conducted to identify all epidemiological and/or clinical studies of obesity in sub-Saharan Africa published in English or French from 1947 to 30 September 2017. A combination of Medical Subject Headings (MeSH) and key word terms used to search the database like 'obese', 'obesity', 'overweight', 'body mass index', 'body fat', 'adiposity', 'body composition', 'body weight', 'percent body fat', 'body fat distribution', 'Africa', 'East Africa', 'Southern Africa', 'Central Africa', 'Western Africa', 'children' and 'adolescent' will be used as a combination of free text and thesaurus terms to search for eligible articles to include in the analysis (for more details see online supplementary appendix 1). The search will also include cross-reference lists of identified original articles and reviews for other relevant articles. We will not exclude any articles on the basis of publication date and only human studies in English and French will be included. Data abstraction will be performed from 1 May 2017 to 30 July 2017. Authors will be contacted to provide additional details of information on their research depending on the circumstances. Cross-references of relevant full-text articles will be hand-searched for further original papers.

\section{Study selection for inclusion in the review}

Studies obtained from the literature search will be checked by title and citation. References from the selected studies will also be assessed to ensure that relevant studies are not missed. Studies will be required to meet the following inclusion criteria: reports of original studies written in English and French evaluating the prevalence of obesity and overweight and studies coming from sub-Sahara African settings. All epidemiological studies of observational (cross-sectional, cohort, case-control) and randomised controlled trials will be included. The papers will also include both community- and institutional-based studies. Studies will be excluded from the analysis for any of the following reasons: duplicate publication of the same study; articles available only in abstract form, letters, reviews, commentaries, editorials, case series with small sample size ( $<30$ participants), studies with no appropriate BMI or BMI z score determination and those studies conducted outside sub-Saharan Africa. The selection of articles for review will be done in three stages: titles alone, abstracts and then full-text articles. The PRISMA flow diagram will be applied to summarise and synthesise the selection procedure and process of the articles ${ }^{11}$. Obesity and overweight will be defined in this study based on a BMI cut-off value from any of the established standard definitions for child overweight and obesity measurement: Centres for Disease Control and Prevention (CDC), ${ }^{12}$ International Obesity Task Force $(\mathrm{IOTF})^{13}$ and WHO. ${ }^{14}$

\section{Protocol and registration}

This systematic review and meta-analysis protocol was registered with the PROSPERO International Prospective Register of Systematic Reviews (PROSPERO) (registration number: CRD42017064942). We will use Meta-analysis Of Observational Studies in Epidemiology (MOOSE) guidelines for reporting ${ }^{15}$ and Preferred Reporting Items for Systematic reviews and Meta-Analyses (PRISMA-P) statement ${ }^{16}$ (see online supplementary appendix 2).

\section{Methodological quality assessment}

Studies will be assessed for quality to be included for analysis. Methodological quality of included articles will be examined according to the Newcastle-Ottawa Scale (NOS). This tool was developed to assess the quality of non-randomised studies. ${ }^{17}$ The previously used panel for quality assessment criteria for studies will be adopted and each paper will be examined for quality, with studies graded as of low, medium or high ${ }^{18}$; only medium- and high-quality studies will be included in the meta-analyses. The pooled prevalence will then be calculated as a weighted mean of all the study estimates. The STROBE checklist will be used to evaluate the quality of reporting in each paper. ${ }^{19}$ 


\section{Data extraction and management}

Data abstraction will be performed by three independent reviewers (SB, DH and KT) using a standard data abstraction form. Where there is a disagreement as to whether the inclusion criteria have been met, the relevant paper will be reviewed and differences will be resolved by consensus and through discussion. The selected studies will be reviewed and the following data extracted: title, authors, year of publication, study design, study setting (rural vs urban; hospital vs community-based), sample size, geographical region (central, eastern, southern and western Africa), obesity/overweight determination methodology, data collection procedures, prevalence and its outcome. In some cases where the study included multiple countries, we will take the finding of the prevalence for each country accordingly.

\section{Statistical analysis}

Epi-data Version 3.1 and STATA Version 12 (STATA Corporation, College Station, Texas, USA) software will be used for data entry and analysis respectively. The description of original studies will be assessed by using frequency and forest plots. The overall effect (pooled estimated effect size) of the prevalence of obesity and overweight will be carried out using the Der Simonian-Laird random effects meta-analysis (random effects model) ${ }^{20}$ and measured as the proportion of obesity (with $95 \% \mathrm{CI}$ ). Furthermore, stratified analysis will be included for rural versus urban settings and childhood versus adult prevalence of overweight/obesity.

\section{Subgroup analysis}

\section{Statistical heterogeneity and publication bias}

A funnel plot will be produced to assess publication bias, with the symmetry of the plot assessed visually and by Begg rank correlation, and Egger weighted regression test methods will be used to statistically assess publication bias $(\mathrm{P}<0.05$ will be considered as indicative of statistically significant publication bias). Cumulative meta-analysis will also be used to see the effect of each study for pooled estimates. Statistical heterogeneity will be assessed by the Cochran's $\mathrm{Q}$ test $(\mathrm{P}<0.10$ considered indicative of statistically significant heterogeneity), which tests whether the amount of between-study heterogeneity is greater than the probability of chance, ${ }^{21}$ and the $\mathrm{I}^{2}$ statistic which assesses the magnitude of statistical heterogeneity that can be expected by partitioning out the chance heterogeneity (values of $25 \%, 50 \%$ and $75 \%$ are considered to represent low, medium and high heterogeneity, respectively). We will use $\tau^{2}$ to estimate the total amount of heterogeneity. ${ }^{22}$

\section{DISCUSSION}

The study will shed light on the understanding of the epidemiology of obesity and overweight in sub-Saharan Africa. It is important to highlight the slowly growing existence of obesity in the global community and in Africa in particular.
As LMICs have shown a transition in nutrition in the recent past, providing evidence on the epidemiology of overweight and obesity will help in the decision-making processes to be taken by nutritionists, public health workers and medical practitioners. While knowing the prevalence, this systematic review includes all target groups and different settings to make a comprehensive and exhaustive review in the area of obesity and overweight.

\section{Ethics and dissemination}

The study adheres to the Declaration of Helsinki. The underlying work is based on systematic reviews of published data, and therefore does not require ethical review approval. The findings of the systematic review will be disseminated at different conferences and seminars and published in a reputable and refereed international peer-reviewed journal.

Contributors SB: conceived and designed the study, literature search, data extraction and analysis and drafted the protocol. TM, DH, BG: conceived and designed the study and participated in the critical revision of the manuscript. YM, KT: conceived and designed the study, literature search, data extraction and analysis, data extraction and took part in the critical revision of the manuscript. All authors read and approved the final manuscript.

\section{Competing interests None declared.}

Provenance and peer review Not commissioned; externally peer reviewed.

Open Access This is an Open Access article distributed in accordance with the Creative Commons Attribution Non Commercial (CC BY-NC 4.0) license, which permits others to distribute, remix, adapt, build upon this work non-commercially, and license their derivative works on different terms, provided the original work is properly cited and the use is non-commercial. See: http://creativecommons.org/ licenses/by-nc/4.0/

(C) Article author(s) (or their employer(s) unless otherwise stated in the text of the article) 2017. All rights reserved. No commercial use is permitted unless otherwise expressly granted.

\section{REFERENCES}

1. Ng M, Fleming T, Robinson M, et al. Global, regional, and national prevalence of overweight and obesity in children and adults during 1980-2013: a systematic analysis for the Global Burden of Disease Study 2013. Lancet 2014;384:766-81.

2. Yarahmadi S, Etemad K, Hazaveh AM, et al. Urbanization and noncommunicable risk factors in the capital city of six big provinces of Iran. Iran J Public Health 2013;42:113-8.

3. GBD 2015 Risk Factors Collaborators. Global, regional, and national comparative risk assessment of 79 behavioural, environmental and occupational, and metabolic risks or clusters of risks, 1990-2015: a systematic analysis for the Global Burden of Disease Study 2015 Lancet 2016;388:1659-724.

4. World Health Organization (WHO). Obesity and overweight. Geneva: World Health Organization, 2016.

5. World Health Organization (WHO). Draft final report of the Commission on Ending Childhood Obesity. Geneva: World Health Organization, 2015.

6. Kelly AS, Barlow SE, Rao G, et al. Severe obesity in children and adolescents: identification, associated health risks, and treatment approaches: a scientific statement from the American Heart Association. Circulation 2013;128:1689-712.

7. Field AE, Cook NR, Gillman MW. Weight status in childhood as a predictor of becoming overweight or hypertensive in early adulthood. Obes Res 2005;13:163-9.

8. Krassas GE, Tzotzas T. Do obese children become obese adults: childhood predictors of adult disease. Pediatr Endocrinol Rev 2004;1:455-9.

9. Ball GD, Mushquash AR, Keaschuk RA, et al. Using intervention mapping to develop the Parents as Agents of Change (PAC) intervention for managing pediatric obesity. BMC Res Notes 2017; 10:43. 
10. Henderson EJ, Ells LJ, Rubin GP, et al. Systematic review of the use of data from national childhood obesity surveillance programmes in primary care: a conceptual synthesis. Obes Rev 2015;16:962-71.

11. Moher D, Liberati A, Tetzlaff J, et al. Preferred reporting items for systematic reviews and meta-analyses: the PRISMA statement. BMJ 2009;339:b2535.

12. Kuczmarski RJ, Ogden CL, Guo SS, et al. 2000 CDC growth charts for the United States: methods and development. Vital Health Stat 11 2002;2002:1-190.

13. Cole TJ, Bellizzi MC, Flegal KM, et al. Establishing a standard definition for child overweight and obesity worldwide: international survey. BMJ 2000;320:1240-3.

14. de Onis M, Onyango AW, Borghi E, et al. Development of a WHO growth reference for school-aged children and adolescents. Bull World Health Organ 2007;85:660-7.

15. Stroup DF, Berlin JA, Morton SC, et al. Meta-analysis of observational studies in epidemiology: a proposal for reporting. Meta-analysis Of Observational Studies in Epidemiology (MOOSE) group. JAMA 2000;283:2008-12.
16. Moher D, Shamseer L, Clarke M, et al. Preferred reporting items for systematic review and meta-analysis protocols (PRISMA-P) 2015 statement. Syst Rev 2015;4:1.

17. Wells BS GA, O'Connell D, Peterson J, et al. The Newcastle-Ottawa Scale (NOS) for assessing the quality of nonrandomized studies in meta-analysis. 2017 www.ohri.ca/programs/clinical_epidemiology/ oxford.asp

18. Stanifer JW, Jing B, Tolan S, et al. The epidemiology of chronic kidney disease in sub-Saharan Africa: a systematic review and metaanalysis. Lancet Glob Health 2014;2:e174-181.

19. Vandenbroucke JP, von Elm E, Altman DG, et al. Strengthening the Reporting of Observational Studies in Epidemiology (STROBE): explanation and elaboration. PLoS Med 2007;4:e297.

20. DerSimonian R, Laird N. Meta-analysis in clinical trials revisited. Contemp Clin Trials 2015;45:139-45.

21. Higgins JP, Thompson SG, Deeks JJ, et al. Measuring inconsistency in meta-analyses. BMJ 2003;327:557-60.

22. Higgins JP, Thompson SG. Quantifying heterogeneity in a metaanalysis. Stat Med 2002;21:1539-58. 